\title{
A new microebulliometer for the measurement of the vapor-liquid equilibrium of ionic liquid systems
}

\author{
Pedro J. Carvalho ${ }^{a}$, Imran Khan $^{a}$, António Morais ${ }^{a}$, José F.O. Granjo ${ }^{\text {b }}$, Nuno M.C. Oliveira ${ }^{\mathrm{b}}$, \\ Luís M.N.B.F. Santos ${ }^{\mathrm{c}}$, João A.P. Coutinho ${ }^{\mathrm{a}, *}$ \\ a Departamento de Química, CICECO, Universidade de Aveiro, Campus Universitário de Santiago, 3810-193 Aveiro, Portugal \\ b Departamento de Engenharia Química, Faculdade de Ciências e Tecnologia, Universidade de Coimbra, Rua Sílvio Lima - Pólo II, 3030-790 Coimbra, \\ Portugal \\ c Centro de Investigação em Química, Departamento de Química e Bioquímica, Faculdade de Ciências da Universidade do Porto, Rua do Campo Alegre, 687, \\ P-4169-007 Porto, Portugal
}

\section{A R T I C L E I N F O}

\section{Article history:}

Received 17 December 2012

Received in revised form 19 April 2013

Accepted 11 June 2013

Available online 19 June 2013

\section{Keywords:}

Vapor-liquid equilibrium

Ionic liquids

Water

Ethanol

Microebulliometer

NRTL

\begin{abstract}
A B S T R A C T
Over the last decade ionic liquids appeared as potential entrainers for extractive distillation processes. However experimental vapor-liquid equilibrium data for ionic liquid containing systems is still scarce since most conventional equilibrium cells are not adequate for these systems. To overcome that limitation a new isobaric microebulliometer, operating at pressures ranging from 0.05 to $0.1 \mathrm{MPa}$ and requiring a sample volume lower than $8 \mathrm{~mL}$ was developed and validated in this work.

The new apparatus was used to determine isobaric VLE data at pressures of $0.05,0.07$ and $0.1 \mathrm{MPa}$ for eight binary mixtures of 1-ethyl-3-methylimidazolium chloride $\left(\left[\mathrm{C}_{2} \mathrm{mim}\right][\mathrm{Cl}]\right), 1$-butyl-3methylimidazolium chloride $\left(\left[\mathrm{C}_{4} \mathrm{mim}\right][\mathrm{Cl}]\right)$, 1-hexyl-3-methylimidazolium chloride $\left(\left[\mathrm{C}_{6} \mathrm{mim}\right][\mathrm{Cl}]\right)$, and choline chloride $\left(\left[\mathrm{N}_{111(2 \mathrm{OH})}\right][\mathrm{Cl}]\right)$ with water and ethanol. The experimental data here measured were correlated with the NRTL model.
\end{abstract}

(c) 2013 Elsevier B.V. All rights reserved.

\section{Introduction}

Distillation is still the most used separation process in the chemical industry and also one of the most energy intensive operations, for which small improvements may turn into large operational and cost advantages. One of the most challenging areas in applying distillation is the separation of azeotropic mixtures. Extractive distillation stands as one of the best approaches to deal with these systems, allowing the entrainer to be kept in the liquid phase from where it is later separated in a secondary distillation and, therefore, be reused. Organic solvents, inorganic salts and hyper-branched polymers have been investigated as entrainers [1]. Recently, ionic liquids (ILs) a novel class of solvents have attracted an increased interest as entrainers for extractive distillation [2].

Ionic liquids (ILs) are salts composed of large organic cations and organic or inorganic anions that cannot form an ordered crystal and thus remain liquid at or near room temperature. They have been reported to have good entrainer characteristics for azeotrope separation [3]. Their negligible flammability and vapor pressure, high thermal stability and solvating capacity for both polar and

\footnotetext{
* Corresponding author. Tel.: +351234 401 507; fax: +351234370 084.

E-mail address: jcoutinho@ua.pt (J.A.P. Coutinho).
}

non-polar compounds confer them several advantages over other entrainers. Moreover, the physical and corrosion properties are more favorable, for separation processes, than those of the commonly used inorganic molten salts or high-branched polymers [3]. Arlt and co-workers [4] first proposed the use of ionic liquids as potential solvents in extractive distillation to separate water-ethanol mixtures. The authors studied imidazolium-based ILs, with chloride and tetrafluoborate anions, and concluded that these solvents are capable of breaking the water-ethanol azeotrope [4]. A recent work by Rebelo's group [3] reviews the publications on azeotrope breaking using ILs, concluding that imidazolium-based ILs with the chloride anion stands as one of the most promising choices, even over the conventional entrainer, 1,2-ethanediol [4], or even compared with the most overall effective, $\mathrm{CaCl}_{2}$ [3]. Nevertheless, and despite the increasing number of publications experimental data is still scarce [5-7], to adequately design or select the optimal ionic liquid among the 106 potential ILs [8]. One major issue that limits the measurement of vapor-liquid equilibrium in systems containing ionic liquids is that the available equipments, developed for conventional solvents, require a large sample volume: Wang et al. [9] reported VLE data measured using an apparatus from Tokyo Rika Kikai Co. with a total volume of $500 \mathrm{~cm}^{3}$ of which about $250 \mathrm{~cm}^{3}$ is occupied by the sample; Zhao et al. [6] used a dual circulation vapor-liquid equilibrium still, with 


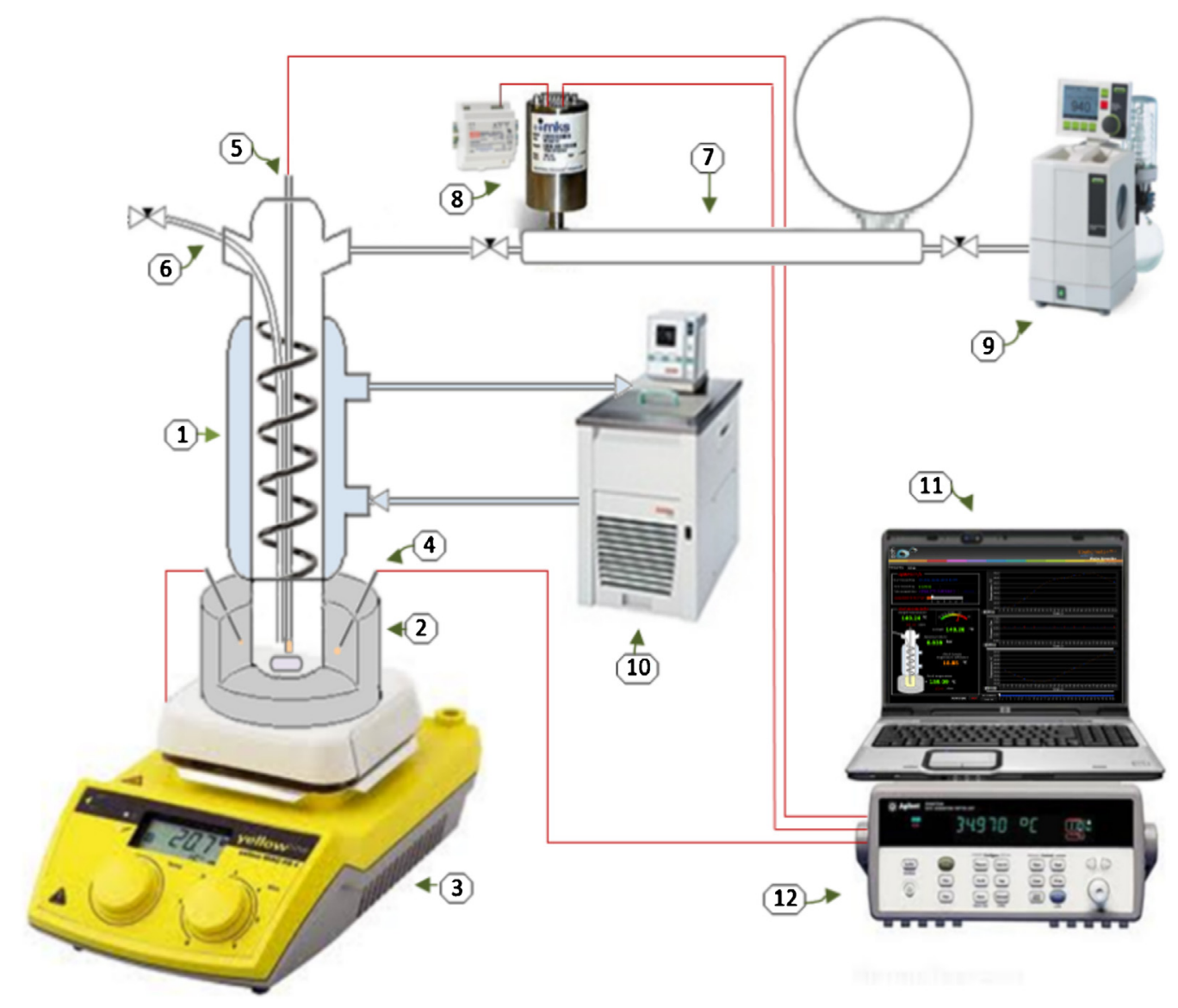

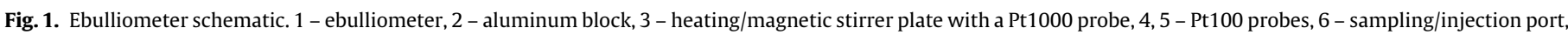

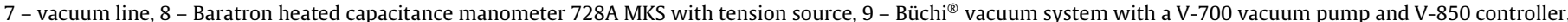
10 - thermostat bath (Julabo F25 MC), 11 - computer to data acquisition, 12 - Agilent 34970A data acquisition/switch unit.

an approximate total volume of the still of $120 \mathrm{~cm}^{3}$, requiring about $80 \mathrm{~cm}^{3}$ of sample; Chen et al. [10] used recirculation VLE still with internal volume of about $120 \mathrm{~cm}^{3}$ of which the liquid occupied about $100 \mathrm{~cm}^{3}$. The VLE cell used by most researchers on this field such as Alvarez et al. [7], Andreatta et al. [11,12] and Calvar et al. $[5,13,14]$ is a glass Fischer Labodest equilibrium still that requires a sample volume of circa $35 \mathrm{~cm}^{3}$. Consequently, measurements of ionic liquid systems using these equipments are quite expensive and the high viscosity of the mixtures rich in ionic liquid difficult a regular boiling of the mixture.

To overcome these limitations a new isobaric ebulliometer able to operate at pressures ranging from 0.05 to $0.1 \mathrm{MPa}$, and requiring a sample volume lower than $8 \mathrm{~cm}^{3}$ was developed, validated and used to determine vapor-liquid equilibria of eight systems of ethanol/water + chloride-based ILs.

\section{Experimental}

\subsection{Materials}

Four ILs based on the chloride anion, 1-ethyl-3-methyl imidazolium chloride $\left(\left[\mathrm{C}_{2} \mathrm{mim}\right][\mathrm{Cl}]\right)$, 1-butyl-3-methylimid azolium chloride $\left(\left[\mathrm{C}_{4} \mathrm{mim}\right][\mathrm{Cl}]\right)$, 1-hexyl-3-methylimidazolium chloride $\left(\left[\mathrm{C}_{6} \mathrm{mim}\right][\mathrm{Cl}]\right)$, and 2-hydroxy- $N, N, N$-trimethylamm onium chloride $\left(\left[\mathrm{N}_{111(2 \mathrm{OH})}\right][\mathrm{Cl}]\right.$, a.k.a. choline chloride), were used in this study. The 1-alkyl-3-methylimidazolium chloride ILs were obtained from Io-li.tec with mass fraction purities higher than $98 \%$. The 2-hydroxy- $N, N, N$-trimethylammonium chloride was obtained from Sigma-Aldrich with mass fraction purity higher than $99 \%$. To reduce to negligible values both water and volatile compounds, high vacuum $\left(10^{-5} \mathrm{mbar}\right)$, stirring, and moderate temperature $(303 \mathrm{~K})$ for a period of at least $48 \mathrm{~h}$ were applied prior to the measurements. The final IL water content was determined with a Mettler Toledo DL32 Karl Fischer coulometer (using the Hydranal - Coulomat E from Riedel-de Haen as analyte), indicating a water mass fraction lower than $30 \times 10^{-6}$. The purity of each ionic liquid was further checked by ${ }^{1} \mathrm{H}$ and ${ }^{13} \mathrm{C}$ NMR.

The ethanol used was obtained from Merck with mass fraction purity higher than 99.8\%. Being highly hygroscopic, the ethanol was kept under low water content through the use of molecular sieves immersed within the compound. Furthermore the ionic liquid, prior to use, was kept under low vacuum $\left(10^{-2} \mathrm{mbar}\right)$. The water used was double distilled and deionized. The decane and $p$ xylene was obtained from Aldrich and Acros Organics respectively, with mass fraction purity higher than $99.8 \%$.

\subsection{Experimental equipment}

A new isobaric ebulliometer able to operate at pressures ranging from 0.05 up to $0.1 \mathrm{MPa}$, was designed, assembled and tested in our laboratory. The ebulliometer, schematically presented in Fig. 1, is composed by three sections: a glass sample chamber container, with a total volume of $8 \mathrm{~mL}$, settled inside of an aluminum block placed on top of an heating/stirring plate; a glass condenser, surrounding the top section of the ebulliometer sample chamber, where the temperature is kept constant by means of a thermostatic bath; a liquid sampling/injection, temperature probe and pressure line connections, done by means of vacuum tide 


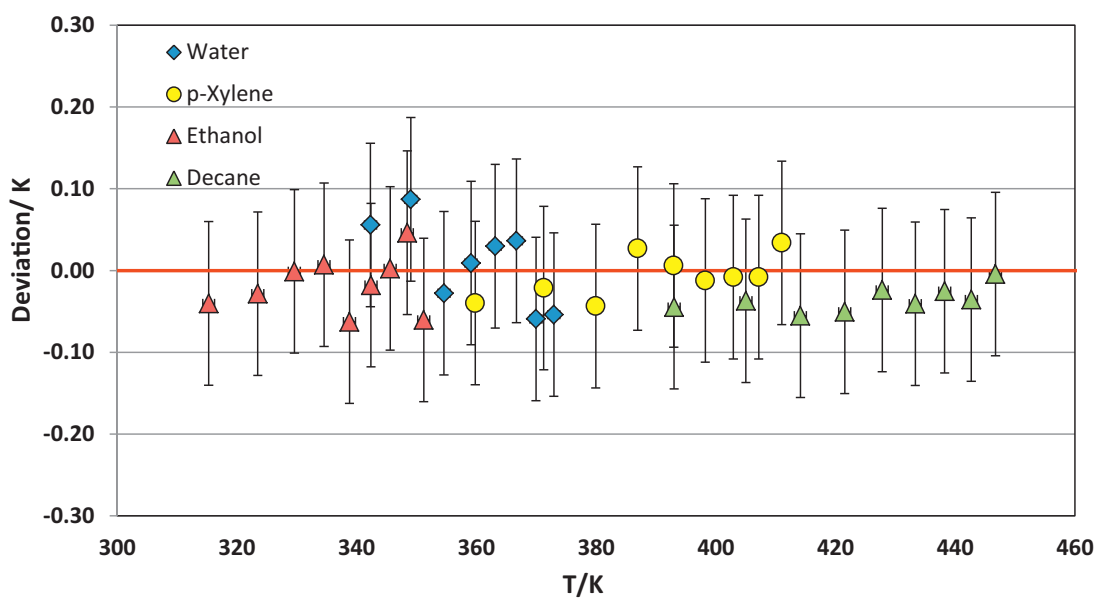

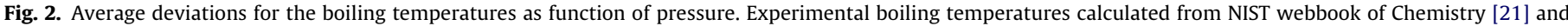
DIPPR's database [22].

teflon sealed ports. Inside the ebulliometer top section a removable glass spiral increases the surface area of the reflux/condenser and the condenser, placed immediately above the sample chamber and connected to a thermostatic bath, assure a better condensation of the vapor phase generated. Some effort was done in the design of this new ebulliometer to have an optimal (low) temperature difference between the evaporation region and the condenser walls in order to establish a regular reflux with a small energy (heat) balance (low recirculation heat and mass flow). This design strategy makes possible the measurement of the equilibrium temperatures in conditions of small fractions of volatile component. The cylindrical aluminum block, with its smooth external wall and the sample chamber placed on its geometrical center, together with the small magnetic stirrer placed inside the ebulliometer sample chamber allows good homogenization of the sample concentration and temperature. The pressure is controlled and kept constant trough a vacuum line, with an internal volume of $5 \times 10^{-3} \mathrm{~m}^{3}$, connected to a Büchi V-700 vacuum pump and V-850 pressure monitoring and controller unit. Pressure measurements are done using a Baratron type capacitance Manometer, MKS model 728A, with temperature regulation at $100{ }^{\circ} \mathrm{C}$ to avoid solvent condensation and with an accuracy of $0.5 \%$. The large volume of the vacuum line allows a better pressure regulation. A metallic sealed Pt100 class A temperature probe placed in the aluminum block, close to the ebulliometer cavity, is used to measures and control the temperature of the heater block. The temperature of the liquid phase inside the ebulliometer is measured by means of a fast response glass sealed Pt100 class $1 / 10$, calibrated previously against a calibrated platinum resistance thermometer, SPRT100 (Fluke-Hart Scientific 1529 Chub-E4), traceable to the National Institute of Standards and Technology (NIST), with an uncertainty less than $2 \times 10^{-2}$.

A mixture rich in ethanol or water is introduced inside the ebulliometer sample chamber and allowed to reach equilibrium, with constant and smooth boiling. Once the equilibrium is reached, the boiling temperature is measured, the liquid phase sampled and the mixture composition determined through an Anton Paar Abbemat 500 Refractometer, with an uncertainty of $2 \times 10^{-5} \mathrm{nD}$, using a calibration curve previously established. This procedure allows the determination of the sample composition within $\pm 0.001 \mathrm{~mol}$ fraction. Subsequently, fixed amounts of IL, water or ethanol are introduced into the ebulliometer, to change the mixture composition, and the procedure is repeated. The IL is kept under moderate vacuum $\left(10^{-2}\right.$ mbar) between measurements, to assure no water absorption from atmosphere.
Since no reference data is available on the literature for these systems, and the amount of available data is quite limited, the adequacy of the apparatus to measure this type of systems is here established using the measurements of vapor-liquid equilibrium data for pure compounds (ethanol, water, $p$-xylene and decane) covering the temperature range of operation of the equipment and the binary systems $\left[\mathrm{C}_{4} \mathrm{mim}\right][\mathrm{Cl}]+\mathrm{H}_{2} \mathrm{O}$, and $\left[\mathrm{C}_{4} \mathrm{mim}\right][\mathrm{Cl}]+$ ethanol $[5]$, and $\left[\mathrm{C}_{6} \mathrm{mim}\right][\mathrm{Cl}]+\mathrm{H}_{2} \mathrm{O}[15]$.

\section{VLE modeling with the NRTL model}

The experimental data gathered was correlated using the NRTL model [16] to predict the non-ideal behavior of the liquid phase solution. This model is based on the local composition concept, to express the effect of the intermolecular forces (short-range) in the non-randomness of the mixtures, using only binary interaction parameters that are estimated from experimental data. For each binary molecular pair $i-j$, the model has three parameters two interaction parameters $\Delta g_{i j}$ and $\Delta g_{j i}$ and the non-randomness parameter $\alpha_{i j}\left(\alpha_{i j}=\alpha_{j i}\right)$. The expressions for the excess Gibbs energy $\left(g^{E}\right)$ and the logarithm of the activity coefficient $\left(\ln \gamma_{i}\right)$ given by the NRTL model can be written as [17]:

$$
\begin{aligned}
& \frac{g^{E}}{R T}=\sum_{i}^{n_{c}} x_{i} \ln \gamma_{i}=\sum_{i}^{n_{c}} \frac{x_{i} L_{i}}{M_{i}} \\
& \ln \gamma_{i}=\frac{L_{i}}{M_{i}}+\sum_{j}^{n_{c}} \frac{x_{j} G_{i j}}{M_{j}}\left(\tau_{i j}-\frac{L_{j}}{M_{j}}\right)
\end{aligned}
$$

$L_{i}=\sum_{k}^{n_{c}} x_{k} \tau_{k i} G_{k i}$

$M_{i}=\sum_{k}^{n_{c}} x_{k} G_{k i}$

$G_{i j}=e^{-\alpha_{i j} \tau_{i j}}$

$\tau_{i j}=\frac{\left(g_{i j}-g_{i i}\right)}{R T}=\frac{\Delta g_{i j}}{R T}, \quad i \neq j$ and $\tau_{i j}=0, i=j$

here $i, j, k \in\{1,2,3\}$ represent the different molecular species in the mixture ( 1 - water, 2 - ethanol, 3 - IL), $n_{c}$ corresponds to the number of components and $x_{i}$ refers to molar fractions. 
Table 1

Vapor-liquid equilibrium data for the $\left[\mathrm{C}_{2} \mathrm{mim}\right][\mathrm{Cl}]+\mathrm{H}_{2} \mathrm{O}$ system at $0.1,0.07$ and $0.05 \mathrm{MPa} .^{\mathrm{a}}$

\begin{tabular}{|c|c|c|c|c|c|c|c|c|c|c|c|c|c|c|c|c|c|}
\hline$x_{\mathrm{H}_{2} \mathrm{O}}$ & $T(\mathrm{~K})$ & $\gamma_{\mathrm{H}_{2} \mathrm{O}}$ & $x_{\mathrm{H}_{2} \mathrm{O}}$ & $T(\mathrm{~K})$ & $\gamma_{\mathrm{H}_{2} \mathrm{O}}$ & $x_{\mathrm{H}_{2} \mathrm{O}}$ & $T(\mathrm{~K})$ & $\gamma_{\mathrm{H}_{2} \mathrm{O}}$ & $x_{\mathrm{H}_{2} \mathrm{O}}$ & $T(\mathrm{~K})$ & $\gamma_{\mathrm{H}_{2} \mathrm{O}}$ & $x_{\mathrm{H}_{2} \mathrm{O}}$ & $T(\mathrm{~K})$ & $\gamma_{\mathrm{H}_{2} \mathrm{O}}$ & $x_{\mathrm{H}_{2} \mathrm{O}}$ & $T(\mathrm{~K})$ & $\gamma_{\mathrm{H}_{2} \mathrm{O}}$ \\
\hline \multicolumn{6}{|c|}{$0.1 \mathrm{MPa}$} & \multicolumn{6}{|c|}{$0.07 \mathrm{MPa}$} & \multicolumn{6}{|c|}{$0.05 \mathrm{MPa}$} \\
\hline 0.998 & 373.66 & 0.970 & 0.749 & 400.00 & 0.541 & 0.998 & 364.02 & 0.966 & 0.780 & 383.59 & 0.615 & 0.998 & 355.58 & 0.959 & 0.806 & 371.07 & 0.661 \\
\hline 0.975 & 374.88 & 0.950 & 0.736 & 401.71 & 0.524 & 0.983 & 364.72 & 0.955 & 0.757 & 387.45 & 0.558 & 0.991 & 355.66 & 0.959 & 0.789 & 373.68 & 0.613 \\
\hline 0.966 & 375.65 & 0.934 & 0.719 & 404.71 & 0.490 & 0.972 & 365.06 & 0.952 & 0.752 & 388.47 & 0.544 & 0.979 & 356.24 & 0.951 & 0.774 & 375.36 & 0.590 \\
\hline 0.955 & 376.19 & 0.928 & 0.713 & 406.30 & 0.473 & 0.955 & 366.46 & 0.922 & 0.746 & 389.39 & 0.532 & 0.970 & 356.56 & 0.949 & 0.756 & 378.59 & 0.539 \\
\hline 0.939 & 377.52 & 0.902 & 0.693 & 409.47 & 0.444 & 0.949 & 366.67 & 0.918 & 0.733 & 391.36 & 0.508 & 0.954 & 357.69 & 0.922 & 0.745 & 379.47 & 0.532 \\
\hline 0.923 & 378.51 & 0.885 & 0.663 & 414.52 & 0.402 & 0.929 & 368.25 & 0.886 & 0.722 & 393.61 & 0.480 & 0.943 & 358.36 & 0.906 & 0.708 & 385.15 & 0.462 \\
\hline 0.909 & 379.96 & 0.853 & 0.654 & 416.30 & 0.388 & 0.907 & 370.05 & 0.849 & 0.713 & 394.65 & 0.472 & 0.927 & 359.36 & 0.886 & 0.698 & 386.47 & 0.449 \\
\hline 0.898 & 381.12 & 0.831 & 0.645 & 418.65 & 0.369 & 0.902 & 370.30 & 0.845 & 0.689 & 398.79 & 0.429 & 0.910 & 360.94 & 0.855 & 0.679 & 389.51 & 0.418 \\
\hline 0.886 & 382.25 & 0.811 & 0.636 & 419.93 & 0.361 & 0.888 & 371.42 & 0.825 & 0.659 & 403.11 & 0.393 & 0.903 & 361.33 & 0.849 & 0.670 & 391.27 & 0.400 \\
\hline 0.868 & 384.12 & 0.778 & 0.625 & 422.01 & 0.348 & 0.877 & 372.64 & 0.800 & 0.651 & 405.98 & 0.366 & 0.892 & 362.22 & 0.828 & 0.653 & 393.93 & 0.377 \\
\hline 0.843 & 387.10 & 0.725 & 0.617 & 425.00 & 0.324 & 0.859 & 374.55 & 0.763 & 0.636 & 409.01 & 0.343 & 0.892 & 362.22 & 0.828 & 0.641 & 395.61 & 0.365 \\
\hline 0.824 & 389.34 & 0.689 & 0.603 & 426.90 & 0.315 & 0.840 & 376.36 & 0.732 & 0.618 & 413.90 & 0.307 & 0.879 & 363.41 & 0.801 & 0.636 & 397.54 & 0.346 \\
\hline 0.813 & 390.14 & 0.681 & 0.592 & 430.32 & 0.294 & 0.832 & 377.78 & 0.703 & 0.598 & 417.70 & 0.284 & 0.866 & 364.44 & 0.783 & 0.618 & 402.00 & 0.310 \\
\hline 0.798 & 392.58 & 0.641 & 0.582 & 431.99 & 0.286 & 0.822 & 378.36 & 0.698 & 0.592 & 418.77 & 0.279 & 0.859 & 365.26 & 0.767 & 0.593 & 407.18 & 0.277 \\
\hline 0.777 & 394.56 & 0.618 & 0.568 & 435.03 & 0.271 & 0.812 & 379.90 & 0.670 & 0.578 & 421.93 & 0.263 & 0.843 & 367.61 & 0.713 & 0.580 & 409.30 & 0.266 \\
\hline 0.763 & 396.80 & 0.587 & & & & 0.793 & 382.37 & 0.631 & & & & 0.826 & 369.65 & 0.675 & & & \\
\hline
\end{tabular}

a Standard uncertainties $x, T$ and $\gamma$ are $0.001,0.02 \mathrm{~K}$ and 0.001 , respectively.

Table 2

Vapor-liquid equilibrium data for the $\left[\mathrm{C}_{4} \mathrm{mim}\right][\mathrm{Cl}]+\mathrm{H}_{2} \mathrm{O}$ system at $0.1,0.07$ and $0.05 \mathrm{MPa} .^{\mathrm{a}}$

\begin{tabular}{|c|c|c|c|c|c|c|c|c|c|c|c|c|c|c|c|c|c|}
\hline$x_{\mathrm{H}_{2} \mathrm{O}}$ & $T(\mathrm{~K})$ & $\gamma_{\mathrm{H}_{2} \mathrm{O}}$ & $x_{\mathrm{H}_{2} \mathrm{O}}$ & $T(\mathrm{~K})$ & $\gamma_{\mathrm{H}_{2} \mathrm{O}}$ & $x_{\mathrm{H}_{2} \mathrm{O}}$ & $T(\mathrm{~K})$ & $\gamma_{\mathrm{H}_{2} \mathrm{O}}$ & $x_{\mathrm{H}_{2} \mathrm{O}}$ & $T(\mathrm{~K})$ & $\gamma_{\mathrm{H}_{2} \mathrm{O}}$ & $x_{\mathrm{H}_{2} \mathrm{O}}$ & $T(\mathrm{~K})$ & $\gamma_{\mathrm{H}_{2} \mathrm{O}}$ & $x_{\mathrm{H}_{2} \mathrm{O}}$ & $T(\mathrm{~K})$ & $\gamma_{\mathrm{H}_{2} \mathrm{O}}$ \\
\hline \multicolumn{6}{|c|}{$0.1 \mathrm{MPa}$} & \multicolumn{6}{|c|}{$0.07 \mathrm{MPa}$} & \multicolumn{6}{|c|}{$0.05 \mathrm{MPa}$} \\
\hline 0.988 & 372.66 & 1.014 & 0.762 & 395.74 & 0.608 & 0.985 & 363.49 & 0.998 & 0.766 & 384.83 & 0.601 & 0.988 & 354.92 & 0.993 & 0.769 & 373.89 & 0.626 \\
\hline 0.976 & 373.55 & 0.995 & 0.753 & 397.15 & 0.589 & 0.976 & 364.38 & 0.975 & 0.757 & 386.57 & 0.574 & 0.976 & 355.92 & 0.965 & 0.751 & 376.21 & 0.589 \\
\hline 0.974 & 374.48 & 0.965 & 0.745 & 397.55 & 0.588 & 0.969 & 364.72 & 0.969 & 0.755 & 386.33 & 0.581 & 0.970 & 356.11 & 0.965 & 0.751 & 376.74 & 0.578 \\
\hline 0.970 & 374.26 & 0.975 & 0.729 & 400.03 & 0.557 & 0.969 & 364.86 & 0.964 & 0.730 & 389.14 & 0.549 & 0.969 & 356.29 & 0.959 & 0.730 & 378.89 & 0.553 \\
\hline 0.964 & 374.67 & 0.968 & 0.706 & 404.19 & 0.507 & 0.966 & 365.21 & 0.954 & 0.705 & 393.19 & 0.499 & 0.966 & 356.50 & 0.955 & 0.705 & 382.70 & 0.503 \\
\hline 0.945 & 374.76 & 0.983 & 0.681 & 408.99 & 0.457 & 0.947 & 365.91 & 0.949 & 0.688 & 396.71 & 0.458 & 0.945 & 357.17 & 0.949 & 0.691 & 386.37 & 0.454 \\
\hline 0.921 & 378.05 & 0.899 & 0.666 & 411.09 & 0.441 & 0.922 & 368.28 & 0.892 & 0.649 & 402.78 & 0.404 & 0.923 & 359.27 & 0.893 & 0.684 & 387.27 & 0.445 \\
\hline 0.901 & 379.17 & 0.885 & 0.664 & 412.40 & 0.425 & 0.900 & 369.76 & 0.866 & 0.646 & 403.13 & 0.401 & 0.901 & 361.06 & 0.856 & 0.668 & 388.50 & 0.437 \\
\hline 0.879 & 380.97 & 0.853 & 0.647 & 415.09 & 0.405 & 0.875 & 371.45 & 0.838 & 0.626 & 405.83 & 0.382 & 0.880 & 362.41 & 0.834 & 0.656 & 391.83 & 0.400 \\
\hline 0.866 & 382.49 & 0.822 & 0.628 & 418.62 & 0.378 & 0.863 & 372.86 & 0.808 & 0.620 & 407.62 & 0.366 & 0.867 & 363.72 & 0.804 & 0.651 & 391.99 & 0.401 \\
\hline 0.842 & 385.00 & 0.777 & 0.625 & 420.09 & 0.365 & 0.841 & 375.23 & 0.762 & 0.605 & 410.88 & 0.341 & 0.844 & 365.28 & 0.778 & 0.635 & 393.77 & 0.389 \\
\hline 0.822 & 387.31 & 0.737 & 0.600 & 424.54 & 0.337 & 0.824 & 377.32 & 0.723 & 0.580 & 414.97 & 0.317 & 0.824 & 367.83 & 0.725 & 0.631 & 394.72 & 0.380 \\
\hline 0.789 & 391.18 & 0.678 & 0.585 & 429.69 & 0.302 & 0.801 & 380.38 & 0.669 & & & & 0.802 & 370.39 & 0.679 & 0.609 & 399.53 & 0.339 \\
\hline 0.779 & 394.25 & 0.623 & & 430.10 & 0.301 & 0.778 & 382.72 & 0.635 & & & & 0.777 & 373.30 & 0.632 & 0.581 & 405.02 & 0.301 \\
\hline
\end{tabular}

a Standard uncertainties $x, T$ and $\gamma$ are $0.001,0.02 \mathrm{~K}$ and 0.001 , respectively.

Table 3

Vapor-liquid equilibrium data for the $\left[\mathrm{C}_{6} \mathrm{mim}\right][\mathrm{Cl}]+\mathrm{H}_{2} \mathrm{O}$ system at $0.1,0.07$ and $0.05 \mathrm{MPa} .^{\mathrm{a}}$

\begin{tabular}{|c|c|c|c|c|c|c|c|c|c|c|c|c|c|c|c|c|c|}
\hline$x_{\mathrm{H}_{2} \mathrm{O}}$ & $T(\mathrm{~K})$ & $\gamma_{\mathrm{H}_{2} \mathrm{O}}$ & $x_{\mathrm{H}_{2} \mathrm{O}}$ & $T(\mathrm{~K})$ & $\gamma_{\mathrm{H}_{2} \mathrm{O}}$ & $x_{\mathrm{H}_{2} \mathrm{O}}$ & $T(\mathrm{~K})$ & $\gamma_{\mathrm{H}_{2} \mathrm{O}}$ & $x_{\mathrm{H}_{2} \mathrm{O}}$ & $T(\mathrm{~K})$ & $\gamma_{\mathrm{H}_{2} \mathrm{O}}$ & $x_{\mathrm{H}_{2} \mathrm{O}}$ & $T(\mathrm{~K})$ & $\gamma_{\mathrm{H}_{2} \mathrm{O}}$ & $x_{\mathrm{H}_{2} \mathrm{O}}$ & $T(\mathrm{~K})$ & $\gamma_{\mathrm{H}_{2} \mathrm{O}}$ \\
\hline \multicolumn{6}{|c|}{$0.1 \mathrm{MPa}$} & \multicolumn{6}{|c|}{$0.07 \mathrm{MPa}$} & \multicolumn{6}{|c|}{$0.05 \mathrm{MPa}$} \\
\hline 0.996 & 373.13 & 0.988 & 0.853 & 383.08 & 0.818 & 0.996 & 363.46 & 0.989 & 0.853 & 372.29 & 0.833 & 0.996 & 354.64 & 0.994 & 0.853 & 362.87 & 0.842 \\
\hline 0.994 & 373.39 & 0.983 & 0.840 & 384.20 & 0.800 & 0.993 & 363.56 & 0.986 & 0.840 & 373.49 & 0.810 & 0.994 & 354.70 & 0.994 & 0.840 & 363.37 & 0.839 \\
\hline 0.991 & 373.66 & 0.978 & 0.836 & 384.59 & 0.794 & 0.991 & 363.74 & 0.983 & 0.829 & 374.67 & 0.788 & 0.991 & 354.83 & 0.992 & 0.832 & 364.28 & 0.818 \\
\hline 0.984 & 373.79 & 0.977 & 0.832 & 385.09 & 0.784 & 0.984 & 363.90 & 0.983 & 0.821 & 375.61 & 0.769 & 0.984 & 355.02 & 0.991 & 0.830 & 364.89 & 0.802 \\
\hline 0.980 & 374.03 & 0.973 & 0.820 & 386.27 & 0.765 & 0.980 & 364.14 & 0.978 & 0.820 & 375.70 & 0.768 & 0.982 & 355.19 & 0.986 & 0.827 & 365.21 & 0.795 \\
\hline 0.976 & 374.33 & 0.967 & 0.813 & 387.08 & 0.752 & 0.975 & 364.45 & 0.971 & 0.819 & 375.83 & 0.765 & 0.975 & 355.42 & 0.984 & 0.806 & 366.01 & 0.792 \\
\hline 0.965 & 374.57 & 0.969 & 0.808 & 388.02 & 0.734 & 0.966 & 364.86 & 0.966 & 0.808 & 376.32 & 0.762 & 0.966 & 356.03 & 0.970 & 0.806 & 366.09 & 0.790 \\
\hline 0.954 & 375.10 & 0.963 & 0.796 & 389.15 & 0.718 & 0.956 & 365.37 & 0.957 & 0.800 & 377.54 & 0.738 & 0.956 & 356.69 & 0.955 & 0.800 & 366.60 & 0.781 \\
\hline 0.945 & 375.57 & 0.956 & 0.787 & 390.07 & 0.705 & 0.949 & 366.01 & 0.943 & 0.786 & 378.83 & 0.718 & 0.950 & 357.06 & 0.947 & 0.786 & 368.16 & 0.750 \\
\hline 0.933 & 376.13 & 0.949 & 0.776 & 391.17 & 0.690 & 0.937 & 366.49 & 0.938 & 0.778 & 379.36 & 0.712 & 0.936 & 357.43 & 0.947 & 0.781 & 369.03 & 0.731 \\
\hline 0.918 & 377.09 & 0.933 & 0.767 & 392.23 & 0.675 & 0.920 & 367.27 & 0.927 & 0.775 & 380.34 & 0.692 & 0.920 & 357.96 & 0.944 & 0.774 & 369.71 & 0.720 \\
\hline 0.912 & 377.80 & 0.917 & 0.758 & 393.39 & 0.659 & 0.912 & 367.94 & 0.912 & 0.761 & 381.53 & 0.677 & 0.912 & 358.91 & 0.918 & 0.761 & 370.34 & 0.716 \\
\hline 0.901 & 378.60 & 0.902 & 0.744 & 394.83 & 0.641 & 0.902 & 368.70 & 0.898 & 0.747 & 382.80 & 0.660 & 0.903 & 359.94 & 0.890 & 0.753 & 371.27 & 0.699 \\
\hline 0.887 & 379.60 & 0.885 & 0.718 & 397.29 & 0.615 & 0.888 & 369.79 & 0.875 & 0.725 & 384.70 & 0.639 & 0.889 & 360.40 & 0.888 & 0.721 & 374.92 & 0.641 \\
\hline 0.879 & 380.37 & 0.870 & 0.694 & 400.21 & 0.582 & 0.880 & 370.33 & 0.866 & 0.699 & 386.81 & 0.617 & 0.881 & 360.93 & 0.878 & 0.702 & 376.29 & 0.627 \\
\hline 0.871 & 381.25 & 0.851 & 0.668 & 403.25 & 0.552 & 0.873 & 370.95 & 0.854 & 0.671 & 391.39 & 0.555 & 0.875 & 361.50 & 0.865 & 0.670 & 381.29 & 0.553 \\
\hline 0.864 & 381.96 & 0.838 & 0.649 & 405.30 & 0.534 & 0.864 & 371.80 & 0.837 & 0.655 & 393.61 & 0.530 & 0.864 & 361.83 & 0.865 & 0.655 & 383.26 & 0.530 \\
\hline
\end{tabular}

a Standard uncertainties $x, T$ and $\gamma$ are $0.001,0.02 \mathrm{~K}$ and 0.001 , respectively. 
Table 4

Vapor-liquid equilibrium data for the $\left[\mathrm{N}_{111(2 \mathrm{OH})}\right][\mathrm{Cl}]+\mathrm{H}_{2} \mathrm{O}$ system at $0.1,0.07$ and $0.05 \mathrm{MPa} .^{\mathrm{a}}$

\begin{tabular}{|c|c|c|c|c|c|c|c|c|c|c|c|c|c|c|c|c|c|}
\hline$x_{\mathrm{H}_{2} \mathrm{O}}$ & $T(\mathrm{~K})$ & $\gamma_{\mathrm{H}_{2} \mathrm{O}}$ & $x_{\mathrm{H}_{2} \mathrm{O}}$ & $T(\mathrm{~K})$ & $\gamma_{\mathrm{H}_{2} \mathrm{O}}$ & $x_{\mathrm{H}_{2} \mathrm{O}}$ & $T(\mathrm{~K})$ & $\gamma_{\mathrm{H}_{2} \mathrm{O}}$ & $x_{\mathrm{H}_{2} \mathrm{O}}$ & $T(\mathrm{~K})$ & $\gamma_{\mathrm{H}_{2} \mathrm{O}}$ & $x_{\mathrm{H}_{2} \mathrm{O}}$ & $T(\mathrm{~K})$ & $\gamma_{\mathrm{H}_{2} \mathrm{O}}$ & $x_{\mathrm{H}_{2} \mathrm{O}}$ & $T(\mathrm{~K})$ & $\gamma_{\mathrm{H}_{2} \mathrm{O}}$ \\
\hline \multicolumn{6}{|l|}{$0.1 \mathrm{MPa}$} & \multicolumn{6}{|c|}{$0.07 \mathrm{MPa}$} & \multicolumn{6}{|c|}{$0.05 \mathrm{MPa}$} \\
\hline 0.985 & 372.46 & 1.021 & 0.859 & 381 & 0.854 & 0.985 & 363. & 0.991 & 0.859 & 3 & 0.829 & 0.985 & 355 & 0.984 & 0.860 & 363.24 & 0.824 \\
\hline 0.985 & 371.97 & 1.043 & 0.806 & 386.80 & 0.766 & 0.982 & 363.47 & 1.001 & 0.809 & 376.75 & 0.751 & 0.982 & 355.15 & 0.990 & 0.810 & 367.70 & 0.740 \\
\hline 0.979 & 372.93 & 1.013 & 0.754 & 392.77 & 0.675 & 0.979 & 363.69 & 0.997 & 0.754 & 382.62 & 0.658 & 0.980 & 355.93 & 0.960 & 0.761 & 372.65 & 0.659 \\
\hline 0.971 & 373.12 & 1.014 & 0.705 & 398.97 & 0.595 & 0.972 & 364.03 & 0.992 & 0.705 & 388.36 & 0.582 & 0.972 & 355.71 & 0.978 & 0.704 & 378.69 & 0.577 \\
\hline 0.955 & 374.00 & 0.999 & 0.670 & 402.54 & 0.562 & 0.959 & 364.72 & 0.978 & 0.670 & 392.82 & 0.531 & 0.959 & 356.36 & 0.966 & 0.671 & 382.75 & 0.527 \\
\hline 0.941 & 375.08 & 0.978 & 0.635 & 407.78 & 0.508 & 0.944 & 365.71 & 0.957 & 0.632 & 398.47 & 0.472 & 0.943 & 357.19 & 0.948 & 0.629 & 388.91 & 0.458 \\
\hline 0.933 & 376.35 & 0.942 & 0.618 & 410.03 & 0.490 & 0.933 & 366.16 & 0.954 & 0.619 & 399.90 & 0.462 & 0.936 & 357.67 & 0.940 & 0.623 & 389.14 & 0.460 \\
\hline 0.923 & 376.33 & 0.953 & 0.614 & 411.12 & 0.477 & 0.924 & 366.91 & 0.935 & 0.612 & 400.27 & 0.462 & 0.924 & 358.26 & 0.929 & 0.619 & 389.79 & 0.453 \\
\hline 0.900 & 378.19 & 0.917 & & & & 0.900 & 368.71 & 0.899 & & & & 0.900 & 359.88 & 0.895 & & & \\
\hline
\end{tabular}

a Standard uncertainties $x, T$ and $\gamma$ are $0.001,0.02 \mathrm{~K}$ and 0.001 , respectively.

Although the NRTL model was not developed to describe the behavior of mixtures containing electrolyte species, it has been applied successfully in systems with ionic liquid solutions $[5,18,19]$. This can be explained by the fact that in ILs the ion charge is usually disperse and the long-range electrostatic forces are weak compared with the short-range intermolecular forces (so that they can be neglected). Therefore, in the present work the liquid solutions were modeled as mixtures of non-dissociated components.

Different approaches have been considered for the parameter estimation problem with the NRTL model, to obtain the best set of parameters that describe the VLE data in systems containing ionic
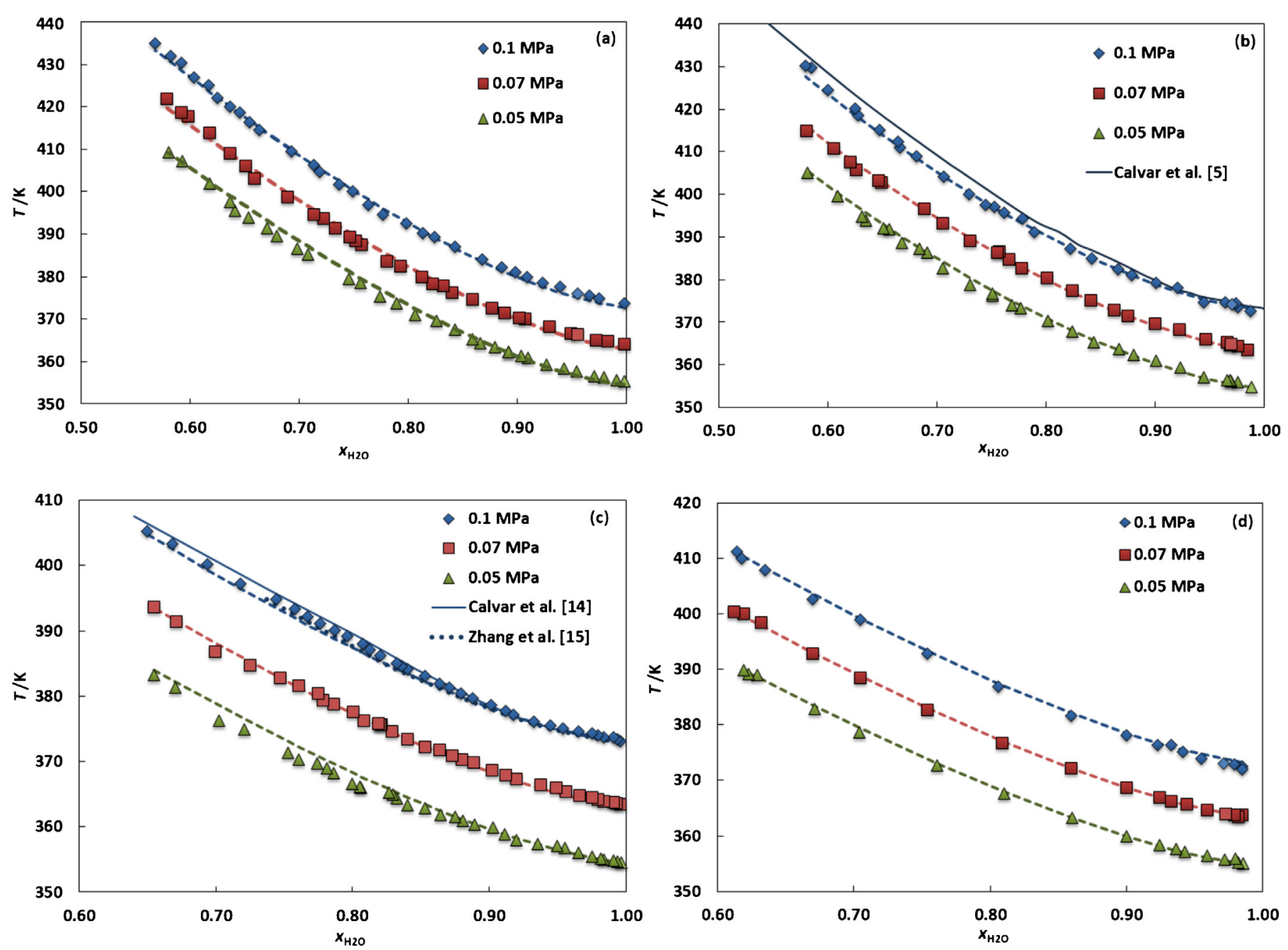

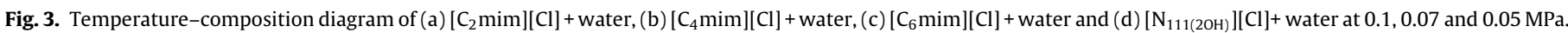
The dashed lines represent the correlation of the data using the NRTL model. liquids. In the present work, this task was formulated as the solution of a nonlinear programming problem (NLP), using the weighted norm of the differences between the solvent (water or ethanol) experimental mole fractions and the values predicted by the model as the objective function:

$\min _{z} \varphi=\sum_{l}^{n t} \omega_{l} e_{l}\left(\Delta g_{i j}\right)^{2}$

In this equation $e_{l}\left(\Delta g_{i j}\right)=x_{l}^{\exp }-x_{l}^{\bmod }\left(\Delta g_{i j}\right)$, where the superscripts exp and mod correspond to the solvent experimental and 
Table 5

Vapor-liquid equilibrium data for the $\left[\mathrm{C}_{2} \mathrm{mim}\right][\mathrm{Cl}]+$ Ethanol system at $0.1,0.07$ and $0.05 \mathrm{MPa}{ }^{\text {a }}$

\begin{tabular}{|c|c|c|c|c|c|c|c|c|c|c|c|c|c|c|c|c|c|}
\hline$x_{\mathrm{EtOH}}$ & $T(\mathrm{~K})$ & $\gamma_{\mathrm{EtOH}}$ & $x_{\mathrm{EtOH}}$ & $T(\mathrm{~K})$ & $\gamma_{\mathrm{EtOH}}$ & $x_{\text {EtOH }}$ & $T(\mathrm{~K})$ & $\gamma_{\text {EtOH }}$ & $x_{\mathrm{EtOH}}$ & $T(\mathrm{~K})$ & $\gamma_{\mathrm{EtOH}}$ & $x_{\mathrm{EtOH}}$ & $T(\mathrm{~K})$ & $\gamma_{\mathrm{EtOH}}$ & $x_{\mathrm{EtOH}}$ & $T(\mathrm{~K})$ & $\gamma_{\mathrm{EtOH}}$ \\
\hline \multicolumn{6}{|c|}{$0.1 \mathrm{MPa}$} & \multicolumn{6}{|c|}{$0.07 \mathrm{MPa}$} & \multicolumn{6}{|c|}{$0.05 \mathrm{MPa}$} \\
\hline 0.997 & 351.10 & 1.002 & 0.759 & 369.56 & 0.662 & 0.997 & 342.66 & 0.986 & 0.700 & 361.14 & 0.678 & 0.995 & 334.80 & 0.989 & 0.707 & 351.22 & 0.703 \\
\hline 0.979 & 351.70 & 0.996 & 0.756 & 369.89 & 0.659 & 0.992 & 342.69 & 0.990 & 0.681 & 361.84 & 0.680 & 0.974 & 335.12 & 1.000 & 0.674 & 353.25 & 0.682 \\
\hline 0.966 & 352.25 & 0.989 & 0.739 & 371.34 & 0.640 & 0.959 & 343.31 & 0.998 & 0.669 & 365.37 & 0.609 & 0.959 & 335.53 & 0.996 & 0.666 & 356.30 & 0.613 \\
\hline 0.950 & 353.07 & 0.974 & 0.720 & 373.03 & 0.619 & 0.939 & 344.05 & 0.987 & 0.640 & 366.33 & 0.614 & 0.939 & 336.07 & 0.989 & 0.643 & 357.71 & 0.601 \\
\hline 0.936 & 353.64 & 0.967 & 0.712 & 375.18 & 0.583 & 0.922 & 344.67 & 0.980 & 0.613 & 369.04 & 0.584 & 0.922 & 336.81 & 0.979 & 0.626 & 360.10 & 0.565 \\
\hline 0.915 & 354.63 & 0.952 & 0.680 & 376.96 & 0.575 & 0.899 & 345.61 & 0.966 & 0.594 & 371.72 & 0.550 & 0.899 & 337.33 & 0.982 & 0.611 & 360.60 & 0.569 \\
\hline 0.891 & 356.24 & 0.918 & 0.654 & 378.99 & 0.559 & 0.872 & 347.14 & 0.936 & 0.560 & 372.53 & 0.567 & 0.872 & 339.07 & 0.940 & 0.604 & 360.13 & 0.585 \\
\hline 0.871 & 357.98 & 0.879 & 0.651 & 381.37 & 0.520 & 0.846 & 348.66 & 0.908 & 0.538 & 373.84 & 0.564 & 0.850 & 340.38 & 0.910 & 0.561 & 363.28 & 0.560 \\
\hline 0.862 & 358.80 & 0.862 & 0.610 & 385.17 & 0.491 & 0.810 & 350.92 & 0.867 & 0.529 & 375.32 & 0.546 & 0.843 & 340.33 & 0.920 & 0.553 & 365.46 & 0.526 \\
\hline 0.836 & 361.07 & 0.817 & 0.610 & 383.87 & 0.511 & 0.807 & 351.30 & 0.859 & 0.517 & 375.97 & 0.547 & 0.811 & 342.63 & 0.869 & 0.533 & 365.88 & 0.538 \\
\hline 0.834 & 360.96 & 0.823 & 0.578 & 388.38 & 0.469 & 0.786 & 353.80 & 0.798 & 0.490 & 378.51 & 0.529 & 0.783 & 345.13 & 0.812 & 0.523 & 368.26 & 0.503 \\
\hline 0.813 & 363.21 & 0.777 & 0.564 & 389.89 & 0.458 & 0.763 & 355.88 & 0.760 & 0.468 & 381.44 & 0.504 & 0.763 & 346.28 & 0.794 & 0.512 & 368.83 & 0.503 \\
\hline 0.798 & 364.46 & 0.757 & 0.537 & 392.85 & 0.440 & 0.750 & 357.97 & 0.714 & & & & 0.753 & 346.88 & 0.785 & 0.478 & 371.58 & 0.491 \\
\hline 0.795 & 365.49 & 0.731 & 0.532 & 392.42 & 0.450 & 0.718 & 358.33 & 0.736 & & & & 0.753 & 346.88 & 0.785 & & & \\
\hline 0.784 & 366.90 & 0.706 & & & & 0.706 & 360.00 & 0.703 & & & & 0.728 & 350.03 & 0.716 & & & \\
\hline
\end{tabular}

a Standard uncertainties $x, T$ and $\gamma$ are $0.001,0.02 \mathrm{~K}$ and 0.001 , respectively.

Table 6

Vapor-liquid equilibrium data for the $\left[\mathrm{C}_{4} \mathrm{mim}\right][\mathrm{Cl}]+$ ethanol system at $0.1,0.07$ and $0.05 \mathrm{MPa}{ }^{\mathrm{a}}$

\begin{tabular}{|c|c|c|c|c|c|c|c|c|c|c|c|c|c|c|c|c|c|}
\hline$x_{\text {EtOH }}$ & $T(\mathrm{~K})$ & $\gamma_{\mathrm{EtOH}}$ & $x_{\text {EtOH }}$ & $T(\mathrm{~K})$ & $\gamma_{\mathrm{EtOH}}$ & $x_{\text {EtOH }}$ & $T(\mathrm{~K})$ & $\gamma_{\mathrm{EtOH}}$ & $x_{\text {EtOH }}$ & $T(\mathrm{~K})$ & $\gamma_{\mathrm{EtOH}}$ & $x_{\text {EtOH }}$ & $T(\mathrm{~K})$ & $\gamma_{\text {EtOH }}$ & $x_{\mathrm{EtOH}}$ & $T(\mathrm{~K})$ & $\gamma_{\mathrm{EtOH}}$ \\
\hline \multicolumn{6}{|c|}{$0.1 \mathrm{MPa}$} & \multicolumn{6}{|c|}{$0.07 \mathrm{MPa}$} & \multicolumn{6}{|c|}{$0.05 \mathrm{MPa}$} \\
\hline 1.000 & 351.07 & 0.999 & 0.749 & 368.11 & 0.706 & 1.000 & 342.76 & 0.980 & 0.780 & 357.23 & 0.707 & 1.000 & 335.03 & 0.972 & 0.736 & 354.35 & 0.598 \\
\hline 0.983 & 351.42 & 1.003 & 0.734 & 371.50 & 0.640 & 0.983 & 342.87 & 0.991 & 0.776 & 356.19 & 0.739 & 0.983 & 335.25 & 0.984 & 0.711 & 356.97 & 0.560 \\
\hline 0.977 & 351.51 & 1.005 & 0.703 & 375.06 & 0.592 & 0.977 & 342.83 & 1.000 & 0.768 & 358.07 & 0.695 & 0.971 & 334.77 & 1.016 & 0.710 & 357.22 & 0.555 \\
\hline 0.951 & 352.63 & 0.986 & 0.681 & 378.76 & 0.541 & 0.963 & 343.25 & 0.997 & 0.734 & 362.81 & 0.610 & 0.959 & 335.55 & 0.997 & 0.683 & 359.18 & 0.536 \\
\hline 0.944 & 352.98 & 0.981 & 0.673 & 379.12 & 0.540 & 0.955 & 343.59 & 0.992 & 0.714 & 365.59 & 0.568 & 0.931 & 336.70 & 0.976 & 0.673 & 361.50 & 0.499 \\
\hline 0.922 & 353.72 & 0.976 & 0.655 & 380.76 & 0.526 & 0.943 & 343.97 & 0.987 & 0.685 & 368.88 & 0.527 & 0.925 & 337.33 & 0.954 & 0.649 & 363.33 & 0.484 \\
\hline 0.917 & 353.45 & 0.992 & 0.647 & 382.60 & 0.502 & 0.925 & 345.17 & 0.959 & 0.669 & 369.74 & 0.522 & 0.887 & 339.37 & 0.910 & 0.642 & 364.63 & 0.468 \\
\hline 0.886 & 356.11 & 0.928 & 0.625 & 385.78 & 0.469 & 0.897 & 346.41 & 0.940 & 0.654 & 372.06 & 0.494 & 0.886 & 339.20 & 0.923 & 0.617 & 367.44 & 0.442 \\
\hline 0.855 & 358.54 & 0.876 & 0.613 & 387.01 & 0.460 & 0.887 & 347.36 & 0.915 & 0.645 & 372.93 & 0.486 & 0.857 & 341.51 & 0.864 & 0.592 & 370.22 & 0.416 \\
\hline 0.848 & 358.64 & 0.881 & 0.601 & 390.06 & 0.427 & 0.861 & 348.76 & 0.891 & 0.624 & 376.51 & 0.445 & 0.849 & 341.57 & 0.869 & 0.579 & 372.04 & 0.398 \\
\hline 0.829 & 361.14 & 0.821 & 0.571 & 395.14 & 0.385 & 0.856 & 349.48 & 0.871 & 0.596 & 381.44 & 0.396 & 0.826 & 343.80 & 0.812 & 0.557 & 374.57 & 0.380 \\
\hline 0.818 & 361.25 & 0.827 & 0.543 & 399.20 & 0.360 & 0.848 & 349.56 & 0.876 & 0.575 & 384.38 & 0.373 & 0.819 & 343.56 & 0.828 & 0.532 & 378.17 & 0.353 \\
\hline 0.796 & 364.39 & 0.758 & 0.542 & 399.37 & 0.359 & 0.825 & 352.12 & 0.814 & 0.544 & & 0.339 & 0.797 & 346.44 & 0.758 & 0.513 & 381.62 & 0.328 \\
\hline 0.781 & 365.12 & 0.753 & 0.522 & 402.03 & 0.345 & 0.819 & 352.01 & 0.824 & 0.519 & 391.89 & 0.327 & 0.779 & 349.33 & 0.689 & 0.482 & 385.12 & 0.312 \\
\hline 0.767 & 367.83 & 0.696 & 0.500 & 405.89 & 0.323 & 0.797 & 354.89 & 0.756 & 0.497 & 394.68 & 0.314 & 0.768 & 351.05 & 0.652 & 0.459 & 388.33 & 0.296 \\
\hline
\end{tabular}

a Standard uncertainties $x, T$ and $\gamma$ are $0.001,0.02 \mathrm{~K}$ and 0.001 , respectively.

calculated mole fraction values, respectively. The summation in this equation is taken over all VLE data points $(l)$ of each binary molecular pair (water-IL or ethanol-IL), and $\omega_{l}$ is a weight factor associated with each error term. The objective function $\varphi$ was minimized by simultaneous determination of all quantities (parameters and model predictions, collectively denoted by $z$ ), subjected to constraints of VLE (Eq. (7)), the NRTL activity coefficient model (Eqs. (1)-(6)), sum of molar fraction restrictions, and magnitude bounds for the model parameters $\Delta g_{i j}$. The $\alpha_{i j}$ parameters in the model were considered constant, with $\alpha_{13}=0.2$ and $\alpha_{23}=0.3$, as suggested by Chapeaux et al. [20]. The weights $\omega_{l}$ were considered to be unitary for all data points available.

\section{Results and discussion}

\subsection{Experimental results}

In the absence of reference data for VLE of ionic liquid containing systems the new microebulliometer developed on this work was validated on the measurement of boiling temperatures of the

Table 7

Vapor-liquid equilibrium data for the $\left[\mathrm{C}_{6} \mathrm{mim}\right][\mathrm{Cl}]+$ Ethanol system at $0.1,0.07$ and $0.05 \mathrm{MPa}$.

\begin{tabular}{|c|c|c|c|c|c|c|c|c|c|c|c|c|c|c|c|c|c|}
\hline$x_{\text {EtOH }}$ & $T(\mathrm{~K})$ & $\gamma_{\mathrm{EtOH}}$ & $x_{\text {EtOH }}$ & $T(\mathrm{~K})$ & $\gamma_{\text {EtOH }}$ & $x_{\mathrm{EtOH}}$ & $T(\mathrm{~K})$ & $\gamma_{\text {EtOH }}$ & $x_{\mathrm{EtOH}}$ & $T(\mathrm{~K})$ & $\gamma_{\mathrm{EtOH}}$ & $x_{\text {EtOH }}$ & $T(\mathrm{~K})$ & $\gamma_{\text {EtOH }}$ & $x_{\mathrm{EtOH}}$ & $T(\mathrm{~K})$ & $\gamma_{\mathrm{EtOH}}$ \\
\hline \multicolumn{6}{|c|}{$0.1 \mathrm{MPa}$} & \multicolumn{6}{|c|}{$0.07 \mathrm{MPa}$} & \multicolumn{6}{|c|}{$0.05 \mathrm{MPa}$} \\
\hline 0.991 & 351.94 & 0.974 & 0.750 & 370.60 & 0.647 & 0.993 & 343.07 & 0.974 & 0.752 & 360.47 & 0.649 & 0.992 & 335.15 & 0.980 & 0.751 & 351.72 & 0.648 \\
\hline 0.974 & 352.21 & 0.981 & 0.730 & 373.20 & 0.608 & 0.970 & 343.26 & 0.989 & 0.734 & 362.56 & 0.615 & 0.971 & 335.30 & 0.991 & 0.733 & 354.48 & 0.596 \\
\hline 0.962 & 352.57 & 0.978 & 0.707 & 376.26 & 0.566 & 0.962 & 343.63 & 0.981 & 0.709 & 365.90 & 0.566 & 0.962 & 335.76 & 0.981 & 0.708 & 357.44 & 0.551 \\
\hline 0.949 & 352.97 & 0.976 & 0.682 & 379.50 & 0.527 & 0.950 & 344.05 & 0.977 & 0.681 & 369.39 & 0.521 & 0.949 & 336.27 & 0.972 & 0.678 & 359.90 & 0.525 \\
\hline 0.932 & 353.90 & 0.959 & 0.658 & 383.21 & 0.484 & 0.933 & 344.87 & 0.962 & 0.656 & 372.70 & 0.481 & 0.932 & 336.96 & 0.960 & 0.657 & 363.86 & 0.468 \\
\hline 0.913 & 354.93 & 0.941 & 0.625 & 388.96 & 0.425 & 0.913 & 345.75 & 0.949 & 0.623 & 377.17 & 0.435 & 0.911 & 337.75 & 0.953 & 0.625 & 367.07 & 0.440 \\
\hline 0.884 & 356.83 & 0.905 & 0.605 & 392.29 & 0.396 & 0.883 & 347.68 & 0.907 & 0.605 & 381.50 & 0.389 & 0.883 & 339.57 & 0.906 & 0.600 & 371.50 & 0.392 \\
\hline 0.858 & 358.79 & 0.865 & 0.588 & 394.60 & 0.380 & 0.866 & 348.82 & 0.882 & 0.578 & 385.14 & 0.363 & 0.866 & 340.62 & 0.885 & 0.574 & 375.08 & 0.362 \\
\hline 0.848 & 359.84 & 0.842 & 0.576 & 396.89 & 0.363 & 0.848 & 349.98 & 0.860 & 0.560 & 389.18 & 0.330 & 0.850 & 341.68 & 0.863 & 0.548 & 379.08 & 0.333 \\
\hline 0.822 & 362.09 & 0.800 & 0.550 & 401.81 & 0.329 & 0.823 & 352.13 & 0.815 & 0.551 & 391.16 & 0.316 & 0.823 & 343.36 & 0.830 & 0.520 & 381.16 & 0.328 \\
\hline 0.808 & 364.19 & 0.753 & 0.521 & 406.86 & 0.302 & 0.808 & 354.04 & 0.770 & 0.521 & 395.70 & 0.291 & 0.808 & 345.20 & 0.784 & 0.502 & 385.72 & 0.292 \\
\hline 0.788 & 366.36 & 0.714 & 0.504 & 411.86 & 0.272 & 0.788 & 356.13 & 0.729 & 0.504 & 399.58 & 0.268 & 0.787 & 347.26 & 0.740 & 0.463 & 389.46 & 0.283 \\
\hline 0.767 & 368.68 & 0.676 & & & & 0.769 & 358.19 & 0.691 & & & & 0.769 & 349.20 & 0.700 & & & \\
\hline
\end{tabular}

\footnotetext{
a Standard uncertainties $x, T$ and $\gamma$ are $0.001,0.02 \mathrm{~K}$ and 0.001 , respectively.
} 
Table 8

Vapor-liquid equilibrium data for the $\left[\mathrm{N}_{111(2 \mathrm{OH})}\right][\mathrm{Cl}]+$ ethanol system at $0.1,0.07$ and $0.05 \mathrm{MPa}{ }^{\mathrm{a}}$

\begin{tabular}{|c|c|c|c|c|c|c|c|c|c|c|c|c|c|c|c|c|c|}
\hline$x_{\mathrm{EtOH}}$ & $T(\mathrm{~K})$ & $\gamma_{\text {EtOH }}$ & $x_{\text {EtOH }}$ & $T(\mathrm{~K})$ & $\gamma_{\mathrm{EtOH}}$ & $x_{\text {EtOH }}$ & $T(\mathrm{~K})$ & $\gamma_{\mathrm{EtOH}}$ & $x_{\mathrm{EtOH}}$ & $T(\mathrm{~K})$ & $\gamma_{\mathrm{EtOH}}$ & $x_{\mathrm{EtOH}}$ & $T(\mathrm{~K})$ & $\gamma_{\mathrm{EtOH}}$ & $x_{\mathrm{EtOH}}$ & $T(\mathrm{~K})$ & $\gamma_{\mathrm{EtOH}}$ \\
\hline \multicolumn{6}{|l|}{$0.1 \mathrm{MPa}$} & \multicolumn{6}{|c|}{$0.07 \mathrm{MPa}$} & \multicolumn{6}{|c|}{$0.05 \mathrm{MPa}$} \\
\hline 0.996 & 351.06 & 0.999 & 0.947 & 352.12 & 1.009 & 0.995 & 342.62 & 0.989 & 0.949 & 343.29 & 1.011 & 0.995 & 334.99 & 0.982 & 0.952 & 335.54 & 1.002 \\
\hline 0.995 & 351.48 & 0.984 & 0.947 & 352.13 & 1.007 & 0.989 & 342.53 & 1.000 & 0.947 & 343.51 & 1.002 & 0.989 & 334.81 & 0.997 & 0.949 & 335.55 & 1.007 \\
\hline 0.990 & 351.21 & 1.000 & 0.944 & 352.69 & 0.992 & 0.981 & 342.66 & 1.002 & 0.947 & 343.37 & 1.010 & 0.981 & 334.88 & 1.001 & 0.944 & 335.80 & 1.002 \\
\hline 0.983 & 351.20 & 1.009 & 0.943 & 352.36 & 1.006 & 0.972 & 343.02 & 0.998 & 0.944 & 343.48 & 1.006 & 0.972 & 335.22 & 0.998 & 0.943 & 335.78 & 1.002 \\
\hline 0.972 & 351.88 & 0.991 & 0.940 & 352.86 & 0.990 & 0.966 & 343.05 & 1.002 & 0.942 & 343.56 & 1.005 & 0.965 & 335.31 & 0.999 & 0.939 & 335.84 & 1.003 \\
\hline 0.967 & 351.62 & 1.008 & 0.935 & 352.89 & 0.994 & 0.963 & 343.19 & 1.000 & 0.939 & 343.87 & 0.997 & 0.963 & 335.52 & 0.994 & 0.935 & 335.86 & 1.006 \\
\hline 0.963 & 351.71 & 1.010 & 0.933 & 352.90 & 0.995 & 0.962 & 343.17 & 1.002 & 0.935 & 343.90 & 1.001 & 0.962 & 335.39 & 0.996 & 0.934 & 335.87 & 1.008 \\
\hline 0.956 & 351.99 & 1.003 & 0.931 & 352.88 & 0.999 & 0.956 & 343.37 & 0.998 & 0.931 & 343.87 & 1.007 & 0.956 & 335.59 & 0.996 & 0.931 & 335.89 & 1.012 \\
\hline 0.951 & 352.17 & 1.006 & 0.926 & 353.06 & 0.994 & 0.953 & 343.27 & 1.007 & 0.925 & 344.03 & 1.005 & 0.952 & 335.61 & 1.001 & 0.924 & 336.00 & 1.010 \\
\hline 0.950 & 352.16 & 1.008 & & & & 0.952 & 343.56 & 0.996 & & & & 0.952 & 335.54 & 1.002 & & & \\
\hline
\end{tabular}

a Standard uncertainties $x, T$ and $\gamma$ are $0.001,0.02 \mathrm{~K}$ and 0.001 , respectively.

pure compounds ethanol, water, $p$-xylene and decane, chosen to cover the temperature range of operation of the ebulliometer comprised between 310 and $450 \mathrm{~K}$. At each pressure at least 5 boiling temperatures were measured and the average value and the corresponding standard deviation are presented. The deviations against the boiling temperatures estimated from the vapor pressure correlations reported at NIST Webbook of Chemistry [21] and the DIPPR database [22] are shown in Fig. 2. An uncertainty on the boiling temperatures of $\pm 0.1 \mathrm{~K}$ was obtained.

Experimental isobaric VLE data of the binary systems $\left[\mathrm{C}_{2} \mathrm{mim}\right][\mathrm{Cl}]+\mathrm{H}_{2} \mathrm{O}, \quad\left[\mathrm{C}_{4} \mathrm{mim}\right][\mathrm{Cl}]+\mathrm{H}_{2} \mathrm{O}, \quad\left[\mathrm{C}_{6} \mathrm{mim}\right][\mathrm{Cl}]+\mathrm{H}_{2} \mathrm{O}$ and $\left[\mathrm{N}_{111(2 \mathrm{OH})}\right][\mathrm{Cl}]+\mathrm{H}_{2} \mathrm{O}$ were measured at $0.1,0.07$ and $0.05 \mathrm{MPa}$, and are reported in Tables $1-4$ and depicted in Fig. 3. The $0.1 \mathrm{MPa}$ isobaric VLE data of $\left[\mathrm{C}_{4} \mathrm{mim}\right][\mathrm{Cl}]+\mathrm{H}_{2} \mathrm{O}$ and $\left[\mathrm{C}_{6} \mathrm{mim}\right][\mathrm{Cl}]+\mathrm{H}_{2} \mathrm{O}$ are here compared with those of Calvar et al. [5], and those of $\left[\mathrm{C}_{6} \mathrm{mim}\right][\mathrm{Cl}]+\mathrm{H}_{2} \mathrm{O}$ are also compared with the data by Zhang et al. [15]. The boiling temperatures measured in this work show a good agreement with the literature data for the more diluted solutions, with percentage absolute average deviations (\%AAD) of $0.28 \%$ and $0.14 \%$ for the Calvar et al. [5] [ $\left.\mathrm{C}_{4} \mathrm{mim}\right][\mathrm{Cl}]+\mathrm{H}_{2} \mathrm{O}$ and $\left[\mathrm{C}_{6} \mathrm{mim}\right][\mathrm{Cl}]+\mathrm{H}_{2} \mathrm{O}$ systems, respectively, and $0.16 \%$ for the Zhang et al. [15] $\left[\mathrm{C}_{6} \mathrm{mim}\right][\mathrm{Cl}]+\mathrm{H}_{2} \mathrm{O}$ system. For systems with higher concentrations of ionic liquid and higher temperatures our data for the $\left[\mathrm{C}_{6} \mathrm{mim}\right][\mathrm{Cl}]+\mathrm{H}_{2} \mathrm{O}$ system are in good agreement with
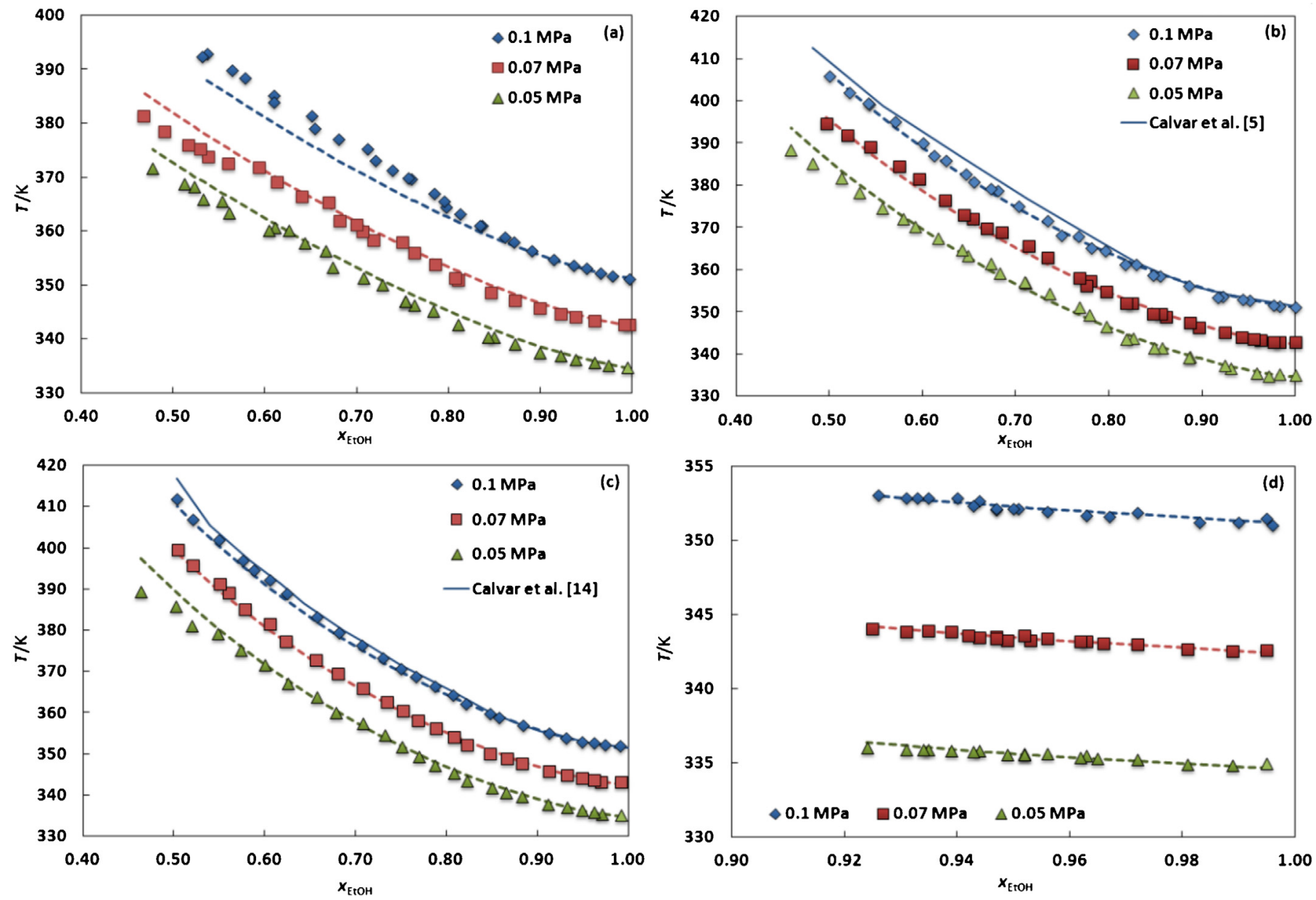

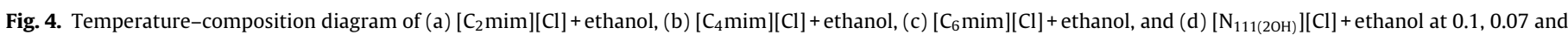
$0.05 \mathrm{MPa}$. The dashed lines represent the correlation of the data using the NRTL model. 

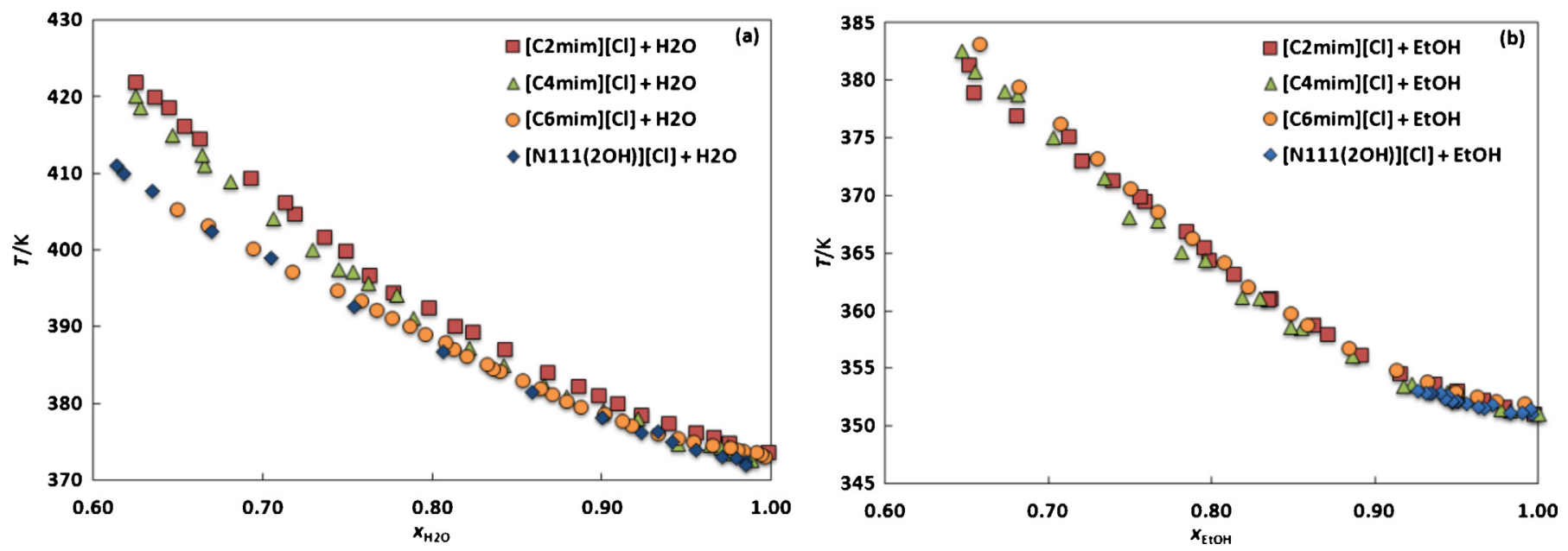

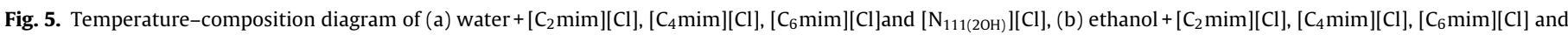
$\left[\mathrm{N}_{111(2 \mathrm{OH})}\right][\mathrm{Cl}]$ at $0.1 \mathrm{MPa}$.

the data by Zhang et al. [15] but some deviations are observed between these two sets of data and the data by Calvar et al. [5] that for both this system and the $\left[\mathrm{C}_{4} \mathrm{mim}\right][\mathrm{Cl}]+\mathrm{H}_{2} \mathrm{O}$ system systematically presents higher boiling temperatures for the higher IL concentrations. These may be due to overheating of the samples due to the difficulty to achieve a regular boiling on a large volume apparatus for these concentrated and viscous solutions.

Experimental isobaric VLE data, at $0.1,0.07$ and $0.05 \mathrm{MPa}$, were also measured for the systems $\left[\mathrm{C}_{2} \mathrm{mim}\right][\mathrm{Cl}]+$ ethanol, $\left[\mathrm{C}_{4} \mathrm{mim}\right][\mathrm{Cl}]+$ ethanol, $\quad\left[\mathrm{C}_{6} \mathrm{mim}\right][\mathrm{Cl}]+$ ethanol and $\left[\mathrm{N}_{111(2 \mathrm{OH})}\right][\mathrm{Cl}]+$ Ethanol in the region of complete miscibility. As reported in Tables 5-8 and depicted in Fig. 4, the mixture boiling temperatures were measured for IL molar fractions up to 0.35 . For the choline chloride its limited miscibility in ethanol restricted the VLE measurements to IL molar fractions below 0.07. Albeit a slight deviation is still observed at high IL concentrations a good agreement between the data reported in this work and those by Calvar et al. [5] for the system $\left[\mathrm{C}_{6} \mathrm{mim}\right][\mathrm{Cl}]+$ ethanol is observed, with a \%AAD of $0.21 \%$. The system [C4mim] $[\mathrm{Cl}]+$ ethanol presents a \%AAD of $0.44 \%$.

An evaluation of the influence of the cation influence on the VLE of the studied systems, presented in Fig. 5, shows some impact of the cation type and alkyl chain length upon the water systems with lower boiling points occurring with increasing alkyl chain length, that reflects the poorer solvation of these cations with longer chains. Curiously, unlike what is observed for the aqueous systems, the ethanol + ILs systems present almost no influence of the cation that could be related with the no differentiation of the solvation of the alkyl chain by the ethanol.

To analyze and compare the interactions water-IL with ethanolIL and evaluate their impact upon the VLE behavior the activity coefficients of the solvents, water and ethanol, were estimated using the following phase equilibrium equation

$\gamma^{i}=\frac{y_{i} \varphi_{i} p}{x_{i} \varphi_{i}^{\sigma} p_{i}^{\sigma}}$

where $p$ and $p^{\sigma}$ are the pressure of the system and the saturation vapor pressure of the pure component $i$ at the system temperature, $x_{i}$ and $y_{i}$ represent the mole fractions of component $i$ in the liquid and vapor phases, $\phi_{i}$ is the fugacity coefficient of component $i$ in the vapor phase, while $\varphi_{i}^{\sigma}$ is the fugacity coefficient of component $i$ in its saturated state. The $\phi_{i}$ and $\varphi_{i}^{\sigma}$ are very close to unit for the systems studied at these low pressures and high temperatures. Since the ionic liquid is non-volatile the vapor phase is only composed of solvent and therefore $y_{i}$ is equal to one. Thus, the solvent activity coefficient in solution can be simplified as

$\gamma^{i}=\frac{p}{x_{i} p_{i}^{\sigma}}$

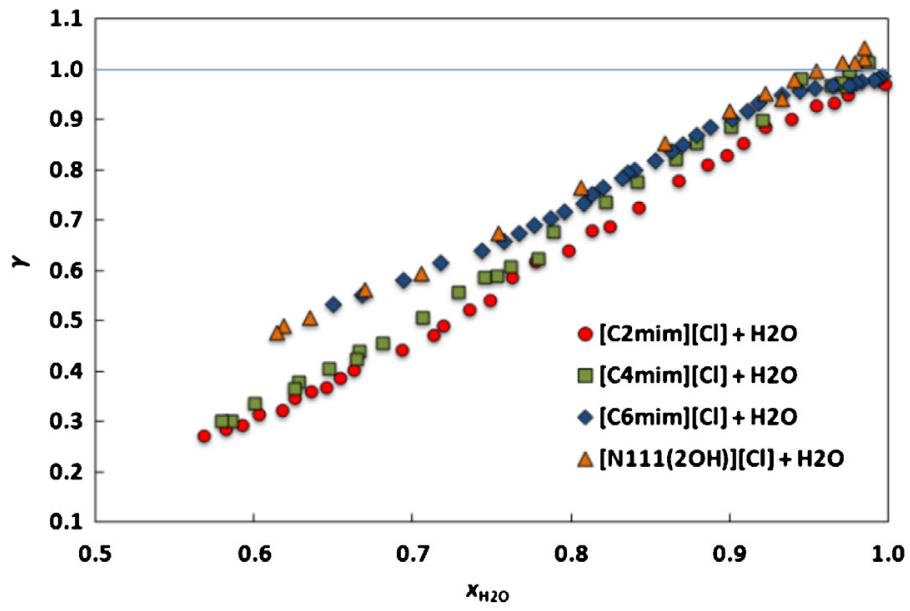

Fig. 6. Activity coefficients as function of water/ethanol mole fractions of the experimental VLE data at $0.1 \mathrm{MPa}$. 
where subscript $i$ refers to the solvent, water or ethanol. The water and ethanol pure component saturation vapor pressures, $p^{\sigma}$, were calculated using correlations obtained from DIPPR's database [22].

The activity coefficients estimated for the studied systems are presented in Fig. 6. It can be observed that all the systems present negative deviations to ideality $(\gamma<1)$, suggesting that the interactions between the ionic liquids and the solvents are favorable. The water + IL systems present activity coefficients that are lower than those for ethanol + IL mixtures denoting, thus, more favorable interactions between the water and the IL than with the ethanol. An almost linear decrease on the activity coefficient of water and ethanol with the increase of the ILs mole fraction is observed between 0.9 and 0.7 mole fraction, in agreement with an increase of the concentration of the ionic liquids and the solvent (water and ethanol) that is an indication of a strong specific interaction of $1: 1$ between the IL and the solvent. It is interesting to suggest that the decrease of the activity coefficient is pointing out to maximum activity for a mole fraction around 0.33 (2 ILs: $1 \mathrm{H}_{2}$ 0/ethanol), indicating the right molecular ratio between the IL and the (H-bond) solvent.

As expected from the boiling temperatures presented in Fig. 4, and confirmed by the activity coefficients of Fig. 6 , the choline chloride, albeit presenting favorable interactions with the water, present interactions clearly less strong than those of imidazolium based ionic liquids. This, along with the very high melting point of this compound and its poor solubility in ethanol suggests that choline chloride is the worst option, among the ionic liquids studied on this work, for breaking the ethanol-water azeotrope.

The strong negative deviations to the ideality, with activity coefficients for equimolar mixtures below 0.5 , are not particular for the systems here reported but can be found in a large number of isobaric VLE systems containing ionic liquids previously reported in the literature $[3,5,7,14]$.

An evaluation of the influence of the pressure on the VLE of the studied systems, presented in Figs. 3 and 4, shows that the pressure drop leads to lower boiling points, as would be expected from Eq. (9). Although the boiling points decrease with the pressure, the same behavior is not observed for the activity coefficients. As depicted in Fig. 7, the differences between the activity coefficients at the various pressures are quite small, often within the experimental uncertainty, what is in good agreement with the excess volumes observed for the systems studied.

Relatively to the numerical solution of the NLP problem in Eq. (2), which often leads to multiple local optimal solutions, this was implemented in GAMS [21], and tackled using the CONOPT [23] and OQNLP [24] solvers. The computer requirements for the parameter estimation task were of the order of one second of CPU for CONOPT and were allowed a maximum of $60 \mathrm{CPU}$ minutes for the runs with the OQNLP solver, on an Intel Xeon 5570 Linux workstation. By taking advantage of the multistart feature of the OQNLP solver, an extensive search for alternative solutions was performed within the computation time established. The values
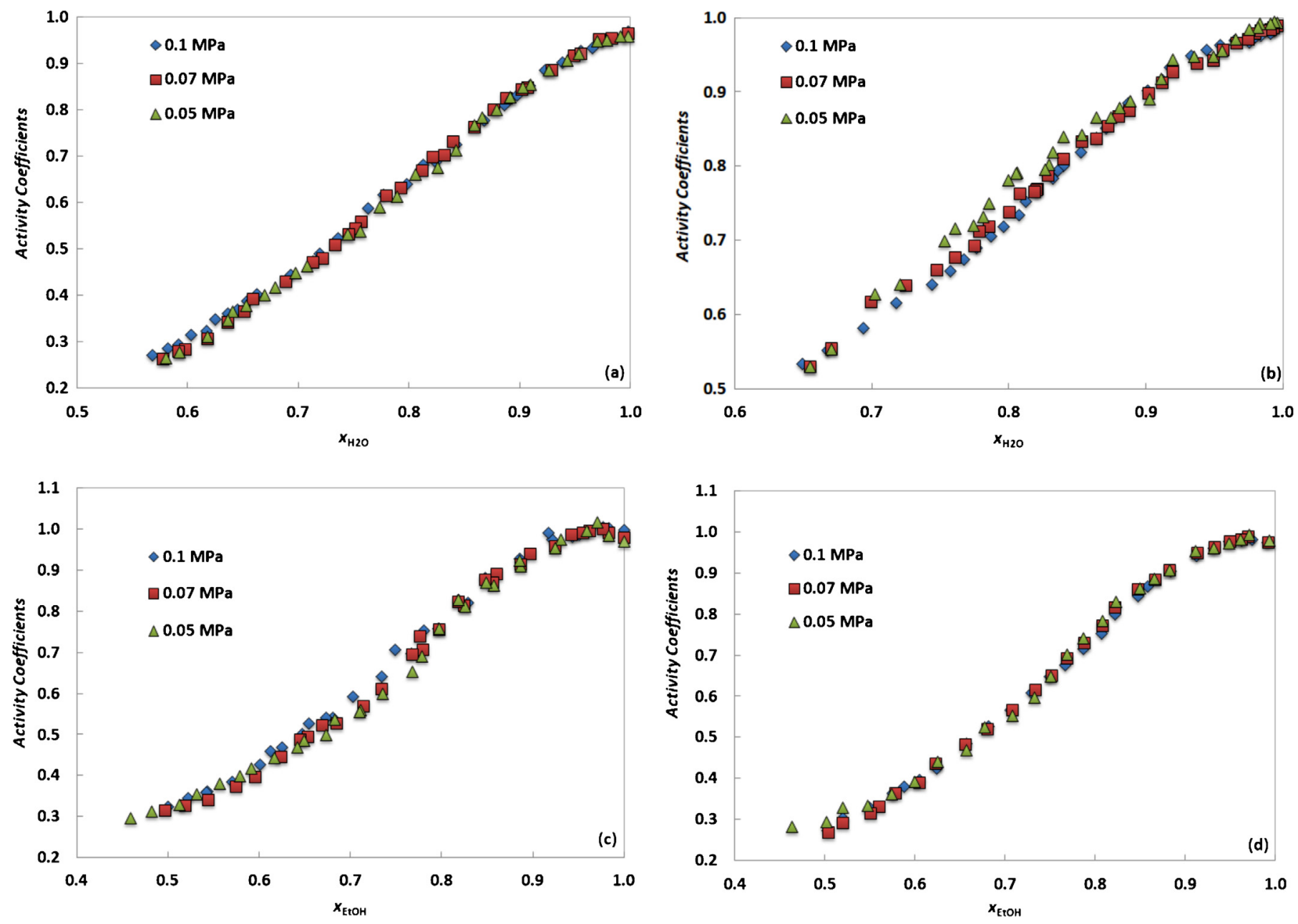

Fig. 7. Activity coefficients for the systems (a) $\left[\mathrm{C}_{2} \mathrm{mim}\right][\mathrm{Cl}]+\mathrm{H}_{2} \mathrm{O}$, (b) $\left[\mathrm{C}_{6}\right.$ mim $][\mathrm{Cl}]+\mathrm{H}_{2} \mathrm{O}$, (c) $\left[\mathrm{C}_{4}\right.$ mim $][\mathrm{Cl}]+$ Ethanol and $(\mathrm{d})\left[\mathrm{C}_{6}\right.$ mim $][\mathrm{Cl}]+\mathrm{ethanol}$. 
Table 9

NRTL model binary interaction parameters $\left(\mathrm{J} \mathrm{mol}^{-1}\right)$ for the systems: 1 - water, 2 ethanol and $3-$ ionic liquid. $\alpha_{13}=0.2$ and $\alpha_{23}=0.3$.

\begin{tabular}{lllll}
\hline Ionic liquid & $\Delta g_{13}$ & $\Delta g_{31}$ & $\Delta g_{23}$ & \multicolumn{1}{c}{$\Delta g_{32}$} \\
\hline$\left[\mathrm{C}_{2} \mathrm{mim}\right][\mathrm{Cl}]$ & -1841.6 & $-11,871$ & 1366.4 & -6543.7 \\
{$\left[\mathrm{C}_{4} \mathrm{mim}\right][\mathrm{Cl}]$} & -4666.2 & $-10,222$ & -5853.7 & -6236.2 \\
{$\left[\mathrm{C}_{6} \mathrm{mim}\right][\mathrm{Cl}]$} & 4318.6 & $-10,435$ & -7638.2 & -6419.8 \\
{$\left[\mathrm{~N}_{111(2 \mathrm{OH})}\right][\mathrm{Cl}]$} & 3961.8 & $-10,718$ & 16,113 & -9128.3 \\
\hline
\end{tabular}

Table 10

Average absolute deviations (\%AAD) in water $\left(x_{\mathrm{H}_{2} \mathrm{O}}\right) /$ ethanol $\left(x_{\mathrm{EtOH}}\right)$ mol fractions for the systems studied at $0.1,0.07$ and $0.05 \mathrm{MPa}$.

\begin{tabular}{lllll}
\hline Ionic liquid & & & & \\
\cline { 3 - 5 } & & 0.1 & 0.07 & 0.05 \\
\hline$\left[\mathrm{C}_{2} \mathrm{mim}\right][\mathrm{Cl}]$ & $\mathrm{H}_{2} \mathrm{O}$ & 0.887 & 0.921 & 1.03 \\
& $\mathrm{EtOH}$ & 2.77 & 1.47 & 1.74 \\
{$\left[\mathrm{C}_{4} \mathrm{mim}\right][\mathrm{Cl}]$} & $\mathrm{H}_{2} \mathrm{O}$ & 0.574 & 0.464 & 0.659 \\
& $\mathrm{EtOH}$ & 0.615 & 0.961 & 1.04 \\
{$\left[\mathrm{C}_{6} \mathrm{mim}\right][\mathrm{Cl}]$} & $\mathrm{H}_{2} \mathrm{O}$ & 0.857 & 0.520 & 0.844 \\
& $\mathrm{EtOH}$ & 0.613 & 0.489 & 0.878 \\
{$\left[\mathrm{~N}_{111(2 \mathrm{OH})}\right][\mathrm{Cl}]$} & $\mathrm{H}_{2} \mathrm{O}$ & 0.273 & 0.241 & 0.426 \\
& $\mathrm{EtOH}$ & 0.640 & 0.471 & 0.473 \\
\hline
\end{tabular}

of the local optima solutions found were recorded and sorted by their quality (decreasing value of $\phi^{*}$ ).

Table 9 summarizes the best set of values that were found for the NRTL parameters of the systems under study. The values of the residual function $\phi$ at the optimum varied from $0.1 \times 10^{-2}$ (water- $\left[\mathrm{N}_{111(2 \mathrm{OH})}\right][\mathrm{Cl}]$ pair) to $7 \times 10^{-2}$ (ethanol- $\left.\left[\mathrm{C}_{2} \mathrm{mim}\right][\mathrm{Cl}]\right)$. The number of local optima found were comprised between 3 (for the pair ethanol- $\left[\mathrm{C}_{2} \mathrm{mim}\right][\mathrm{Cl}]$ ) and 42 (ethanol- $\left[\mathrm{N}_{111(2 \mathrm{OH})}\right][\mathrm{Cl}]$ pair). The solutions delivered by CONOPT corresponded typically to the best local solutions known for each problem. Table 10 lists the average absolute deviation (AAD\%) values in the molar fractions $x_{\mathrm{H}_{2} \mathrm{O}}$ and $x_{\mathrm{EtOH}}$ defined as

$\% \mathrm{AAD}=\left(\frac{100}{N p}\right) \sum_{i=1}^{N p}\left|x_{\text {calc }, i}-x_{\text {exp }, i}\right|$

for each water-IL and ethanol-IL binary systems and pressure datasets. In the $T-x$ binary diagrams for the different ionic liquids in Figs. 1-6, it can be observed that the NRTL model is consistently able to provide a good fit of the experimental data. Nevertheless, it should be noted that the binary parameter values reported here were obtained considering only binary data, and consequently should be used with care in extrapolations to ternary systems and for solvent mole fractions outside the regions where they were correlated.

\section{Conclusions}

A new microebulliometer for the measurement of the boiling temperatures of ionic liquid systems was developed and validated with success. It allows the measurement of VLE data for solutions of ionic liquids in volatile solvents using small quantities of ionic liquids.

Boiling temperatures for systems of four chloride containing ionic liquids with water and ethanol are reported, with six of these systems reported for the first time in the open literature. The results show that the activity coefficients for these systems are temperature dependent, suggesting that these systems present positive excess enthalpies.

The VLE parameters in the NRTL model for water-IL and ethanol-IL binary pairs for four different ionic liquids were estimated by minimizing a weighted sum of squared residuals of the molar fractions of the solvent (water or ethanol). A good fit of the experimental data was obtained, which makes the NRTL model suitable to support the preliminary design of extractive distillation schemes, considering these ILs as potential entrainers.

\section{Acknowledgments}

The authors are thankful for financial support from Fundação para a Ciência e a Tecnologia (Project PTDC/EQUFTT/102166/2008), Laboratório Associado Centro de Investigação em Materiais Cerâmicos e Compósitos (Project Pest-C/CTM/LA0011/2011), the Doctoral grant (SFRH/BD/64338/2009) of José F.O. Granjo and Post-Doctoral grants (SFRH/BPD/82264/2011 and SFRH/BPD/76850/2011) of Pedro J. Carvalho and Imran Khan, respectively.

\section{References}

[1] M. Seiler, D. Kohler, W. Arlt, Sep. Purif. Technol. 29 (2002) 245-263.

[2] Z. Lei, C. Li, B. Chen, Separ. Purif. Rev. 32 (2003) 121-213.

[3] A.B. Pereiro, J.M.M. Araújo, J.M.S.S. Esperança, I.M. Marrucho, L.P.N. Rebelo, J. Chem. Thermodyn. 46 (2012) 2-28.

[4] M. Seiler, C. Jork, A. Kavarnou, W. Arlt, R. Hirsch, AlChE J. 50 (2004) 2439-2454

[5] N. Calvar, B. González, E. Gómez, Á. Domínguez, J. Chem. Eng. Data 51 (2006) $2178-2181$.

[6] J. Zhao, C.C. Dong, C.X. Li, H. Meng, Z.H. Wang, Fluid Phase Equilib. 242 (2006) $147-153$.

[7] V.H. Alvarez, S. Mattedi, M. Aznar, J. Chem. Thermodyn. 43 (2011) 895-900.

[8] N.V. Plechkova, K.R. Seddon, Chem. Soc. Rev. 37 (2008) 123-150.

[9] J. Wang, D. Zheng, L. Fan, L. Dong, J. Chem. Eng. Data 55 (2010) 2128-2132.

[10] R. Chen, L. Zhong, C. Xu, J. Chem. Eng. Data 57 (2012) 155-165.

[11] A.E. Andreatta, M. Francisco, E. Rodil, A. Soto, A. Arce, Fluid Phase Equilib. 300 (2011) 162-171.

[12] A. Arce, J. Martínez-Ageitos, A. Soto, Fluid Phase Equilib. 122 (1996) 117-129.

[13] N. Calvar, B.A. González, E. Goǐmez, A.N. Domiǐnguez, J. Chem. Eng. Data 54 (2009) 1004-1008.

[14] N. Calvar, B. González, E. Gómez, A. Domínguez, Fluid Phase Equilib. 259 (2007) 51-56.

[15] L.Z. Zhang, Y. Ge, D.X. Ji, J.B. Ji, J. Chem. Eng. Data 54 (2009) 2322-2329.

[16] H. Renon, J.M. Prausnitz, AlChE J. 14 (1968) 135-144.

[17] J.P. O'Connell, J.M. Haile, Thermodynamics: Fundamentals for Applications, Cambridge University Press, New York, 2005.

[18] R. Kato, M. Krummen, J. Gmehling, Fluid Phase Equilib. 224 (2004) 47-54.

[19] J. Zhao, X.-C. Jiang, C.-X. Li, Z.-H. Wang, Fluid Phase Equilib. 247 (2006) 190-198.

[20] A. Chapeaux, L.D. Simoni, T.S. Ronan, M.A. Stadtherr, J.F. Brennecke, Green Chem. 10 (2008) 1301-1306.

[21] P.J. Linstrom, W.G. Mallard (Eds.), NIST Chemistry WebBook, NIST Standard Reference Database Number 69, National Institute of Standards and Technology, Gaithersburg, MD, 2012 http://www.webbook.nist.gov

[22] DIPPR 801 Thermophysical Property Database and DIADEM Predictive Software, 2000.

[23] A.S. Drud, J. Comput. 6 (1992) 207-216.

[24] O.a.M.S.D. GAMS Corporation, <http://www.gams.com/dd/docs/solvers/oqnlp. pdf>, 2010 